\title{
Medición de nitratos y nitritos en agua y saliva de población rural precordillerana de la VIII región
}

\author{
Nitrate and nitrite in water and saliva of rural population \\ in the Andes foothills of the VIII Region
}

Thomas Schmidt P1, Sahba Sedaghat N1', Pablo Rosel P², Jorge Fierro ${ }^{3}$.

\section{RESUMEN}

Introducción: Un factor de riesgo para las neoplasias escamosas de faringe son las nitrosaminas, las que derivan de los nitratos salivales que provienen de la dieta y en especial del agua si los niveles están por sobre la norma.

objetivos: Conocer y comparar los niveles de nitratos y nitritos en agua y saliva de habitantes de la región precordillerana y en población urbana de Concepción.

Material y método: Estudio prospectivo, tipo caso y control. Determinan niveles de nitratos y nitritos en agua de la zona precordillerana y en agua de Concepción y en saliva de habitantes de la zona rural y de grupo control de Concepción.

Resultados: Se obtienen 10 muestras de agua del sector rural y 1 muestra de agua potable de Concepción. Nivel de nitratos en agua rural 23,29 mg/L, en agua urbana 3,6 $\mathrm{mg} / \mathrm{L}(p<0,05)$. En saliva, grupo estudio $(n=15)$ con nivel de nitritos de $9,58 \mathrm{mg} / \mathrm{ml}$, grupo control $(n=24)$ nivel de $3,8 \mathrm{mg} / \mathrm{ml}(p<0,05)$.

Conclusión: En la zona rural hay niveles significativamente mayores de nitratos en el agua y nitritos en saliva. Los niveles en agua son superiores a normas nacionales e internacionales. Nuestros resultados resaltan la posible importancia de las nitrosaminas y sus precursores como elementos carcinogónicos en los carcinomas escamosos orofaríngeos.

Palabras clave: Nitritos, nitratos, agua potable, saliva.

\begin{abstract}
Introduction: A risk factor for squamous carcinomas of the oropharynx are the nitrosamines, which are derived from salivary nitrate coming from diet and water, especially if the levels are above the allowed levels.

Aim: To determine and compare the levels of nitrates and nitrites in drinking water and saliva of people at the foothills of our region and urban population of Concepcion.

- Fuente de apoyo: Este trabajo contó con el apoyo financiero de la Sociedad Chilena de Otorrinolaringología, Medicina y Cirugía
\end{abstract}

1 Médico Otorrinolaringólogo, Universidad de Concepción.

2 Residente Otorrinolaringología, Hospital Guillermo Grant Benavente.

Médico Cirujano, Hospital de Coronel. de Cabeza y Cuello. 
Material and method: A descriptive cross sectional study. Determination of levels of nitrates and nitrites in drinking water in the Andean foothills and Concepcion and in saliva of people from same locations.

Results: We obtained 10 rural water samples and 1 from Concepcion drinking water. Nitrate levels in rural water were $23.29 \mathrm{mg} / \mathrm{L}$ and $3.6 \mathrm{mg} / \mathrm{L}(p<0.05)$ in urban water. In saliva, the study group $(n=15)$ had $9.58 \mathrm{mg} / \mathrm{ml}$ nitrite in saliva, the control group ( $n$ =24) $3.8 \mathrm{mg} / \mathrm{ml}(p<0.05)$.

Conclusion: In rural areas there are significantly higher levels of nitrates in drinking water and saliva. The water levels are higher than national and international standards. Our results confirm high amounts of nitrogen cycle products (nitrites and nitrates) in drinking water and in saliva so it could be considered as carcinogenic elements in oropharyngeal squamous cell carcinomas.

Key words: Nitrites, nitrates, water, saliva.

\section{INTRODUCCIÓN}

Los carcinomas escamosos de la cavidad oral y faringe constituyen un porcentaje importante de las neoplasias de manejo por el otorrinolaringólogo. Se caracterizan por presentar una incidencia creciente en población joven, una alta tasa de morbilidad y tasas de mortalidad de hasta $50 \%$ a 5 años ${ }^{1}$.

Uno de los compuestos estudiados como factor de riesgo en relación a estas neoplasias son las nitrosaminas, las que han probado favorecer la carcinogénesis oral a partir de lesiones leucopláquicas por medio del daño al $\mathrm{ADN}^{2}$. La producción de nitrosaminas y su metabolismo a nivel de la cavidad oral se relaciona íntimamente con la absorción de nitratos presentes en la dieta desde el tracto gastrointestinal y a su concentración por las glándulas salivales por medio de un transportador $^{3}$. En la cavidad oral, los nitratos $\left(\mathrm{NO}_{3}\right)$ son transformados en nitritos $\left(\mathrm{NO}_{2}\right)$, los que reaccionan con aminas y amidas para formar nitrosaminas ${ }^{4,5}$.

La saliva juega un rol fundamental en lo que es la carcinogénesis en la cavidad oral, pudiendo las alteraciones oxidativas, producto de especies reactivas del nitrógeno, en la forma de nitrosaminas, llevar a un consumo del sistema antioxidante de la saliva que constituye nuestra primera línea de defensa. Esto puede explicar el daño oxidativo al ADN, con el consiguiente daño a genes supresores y aumento de expresión de protooncogenes que finalmente lleva al cáncer escamoso de cavidad oral ${ }^{6,7}$.

La carcinogenicidad de las nitrosaminas puede resultar de una exposición de corta duración a una dosis elevada 0 a exposición crónica a dosis relativamente bajas. En algunos casos, consumo a largo plazo de agua contaminada con concentraciones menores de nitratos a los establecidos por los estándares internacionales pueden estimular la formación endógena de nitrosaminas ${ }^{8}$.

A pesar que las carnes y los vegetales contienen concentraciones altas de nitratos, el agua bebible es la forma más directa y rápida de consumo de nitratos por la población. Esto se debe a una mayor biodisponibilidad de los nitratos del agua en comparación a los de otros alimentos ${ }^{9}$ especialmente si sus niveles están por sobre la norma de $10 \mu \mathrm{g} / \mathrm{ml}$.

En la VIII región, los carcinomas escamosos de cavidad oral y lengua, constituyen un motivo no infrecuente de derivación hacia el Servicio de Otorrinolaringología del Hospital Regional de Concepción. En una revisión de nuestra casuística entre los años 2000 a 2007 encontramos que provenían predominantemente de comunas rurales de la precordillera de la VIII región con una incidencia de 5,94 por 100.000 habitantes versus 3,74 por 
100.000 habitantes para los del resto de las comunas del mismo período.

Una de las principales actividades económicas de esta región rural es la agricultura y dentro de los productos cultivados se encuentran los cereales lo que no ocurre en las otras zonas rurales de la misma región. La fertilización realizada generalmente consiste en nitrificación del suelo al momento de siembra y en primavera, en dosis elevada, método prohibido en otros países por dejar niveles extremadamente elevados de compuestos nitrogenados (nitratos) en el suelo y que ha causado acumulación importante de productos nitrogenados en las napas de agua superficial y en las cuencas hidrológicas en distintas partes del mundo $0^{10}$.

\section{OBJETIVO}

Postulamos que los nitratos provenientes de los fertilizantes usados en la agricultura contaminan las napas de aguas superficiales que son la principal fuente de agua potable de las zonas rurales de nuestra región, acarreando niveles elevados de nitratos y nitritos en la saliva de los habitantes.

\section{MATERIAL Y MÉTODO}

El presente es un trabajo de investigación de tipo descriptivo de corte transversal. La investigación se realizó mediante la recolección de muestras de agua de pozos superficiales usados para el consumo domiciliario en hogares de zonas rurales precordilleranas de la VIII región (zona de la comuna de Coihueco y de San Carlos) y de agua potable de la comuna de Concepción. En esta ciudad se obtuvo una sola muestra de agua dado que toda el agua potable de la comuna proviene de una misma fuente que es el río Bío-Bío y es procesada en una única planta de tratamiento.

De las residencias rurales cuyos pozos fueron muestreados se obtuvieron muestras de saliva de los habitantes del lugar, al igual que de un grupo control de Concepción, previa realización de consentimiento informado.

El grupo de estudio comprendió pacientes sin antecedentes de patología preneoplásica y neoplá- sica de la cavidad oral y faringe. El grupo control fue determinado mediante pareamiento una vez que se tuvo el grupo de estudio determinado.

Las muestras de agua y saliva fueron sometidas a análisis bioquímico para determinar niveles de nitratos y nitritos. La saliva fue analizada mediante el método descrito por Griess y modificado por Fiddlercon posterior análisis colorimétrico. Los niveles de nitritos en agua fueron determinados por la formación de un producto azo por un complejo diazotado ácido sulfónico con N-1-naftiletilen-diamina y posterior análisis colorimétrico y los de nitratos por medio de la reducción con cadmio metálico para posteriormente formar un compuesto aso analizado en forma colorimétrica. Las determinaciones de los niveles de nitratos y nitritos en agua fueron realizadas en Servicio y Asistencia Tecnológica de la Universidad de Concepción, mientras que el análisis de las muestras de saliva se realizaron en la Facultad de Bioquímica de dicha institución.

El análisis estadístico incluyó cálculo de rangos y promedios para las variables continuas, junto con las pruebas de significancia estadística correspondientes para medir diferencias entre el grupo de estudio y el grupo control y pruebas de correlación para determinar relación significativa entre los niveles de productos nitrogenados en el agua y en la saliva de los sujetos estudiados y del grupo control.

Se determinarán además otras variables consideradas como factores de riesgo para el desarrollo de neoplasias escamosas de cavidad oral y faringe como lo son el consumo de tabaco y alcohol.

\section{RESULTADOS}

Se obtuvieron 10 muestras de agua de pozos superficiales de hogares del sector rural y 1 muestra de agua potable de Concepción. Los resultados de nitratos medidos en agua rural fueron en promedio $23,29 \mathrm{mg} / \mathrm{L}$, con un rango entre 11,3 a $70,5 \mathrm{mg} / \mathrm{L}$, siendo el valor en agua urbana de $3,6 \mathrm{mg} / \mathrm{L}$. Esta diferencia fue estadísticamente significativa ( $p$ $<0,05)$. No se detectaron niveles de nitritos en el agua rural por lo que no se midieron los niveles de éstos en el agua urbana (Tabla 1).

Se analizaron muestras de saliva provenientes de 39 personas divididos en un grupo de población 
Tabla 1. Niveles de nitratos en agua rural y urbano

\begin{tabular}{|cccc|}
\hline & Rural & Urbano & $\mathrm{p}$ \\
\hline Nitratos (N03) & $11,3 \mathrm{mg} / \mathrm{lt}-70,5 \mathrm{mg} / \mathrm{lt} X_{-}: 23,29$ & $3,6 \mathrm{ml} / \mathrm{tt}$ & $\mathrm{p}<005$ \\
\hline
\end{tabular}

rural, con 15 muestras y el grupo control urbano con 24 muestras. En cuanto a su caracterización demográfica, el grupo rural presentó un promedio de edad de 48,2 años, con rango 17 y 71 años. El grupo de población urbano correspondió a 24 pacientes con un rango etario de 19 a 96 años, con promedio de 47,8 años (Tabla 2). La distribución por sexo fue similar, con un predominio masculino de $60 \%$ en el grupo de estudio y $63 \%$ en el grupo control.

En relación al consumo de tabaco y alcohol, el grupo rural presentaba sólo un paciente fumador, que representa $6,6 \%$ de la muestra; mientras que en el grupo control existían 6 pacientes (25\%) con este hábito. Con respecto al consumo de alcohol en el grupo rural y urbano, todos referían un consumo excepcional, definido por una ingesta ocasional en cantidad limitada (1 ó 2 bebidas) y en situaciones muy especiales (menos de 5 veces al año), con $40 \%$ y $41,6 \%$ de prevalencia, respectivamente.

Los resultados de la medición de nitratos y nitritos en saliva se puede observar en las Tablas 3 y 4.

Al separar la población de estudio según sus factores de riesgo asociados, tabaco y consumo de alcohol, no se encontraron diferencias significativas $(p>0,05)$ en los niveles de nitritos y nitratos en

Tabla 2. Caracterización grupo estudio y control

\begin{tabular}{|lll|}
\hline & Grupo estudio (15) & Grupo control (24) \\
\hline Género & & \\
M/F & $9(60 \%) / 6$ & $15(63 \%) / 10$ \\
Edad & 48,2 años $\pm 17,5$ años & 47,8 años $\pm 19,9$ años \\
OH & $6(40 \%)$ & $10(41,6 \%)$ \\
Tabaquismo & $1(6,6 \%)$ & $6(25 \%)$ \\
\hline
\end{tabular}

Tabla 3. Niveles de nitritos en saliva población rural y urbana

\begin{tabular}{|lccc|}
\hline & Promedio & Desviación estándar & $\mathrm{p}$ \\
\hline Rural & $9,58 \_\mathrm{g} / \mathrm{ml}$ & $16,1 \_\mathrm{g} / \mathrm{ml}$ & $<0,033$ \\
Urbana & $3,8 \_\mathrm{g} / \mathrm{ml}$ & $6,6 \_\mathrm{g} / \mathrm{ml}$ & \\
\hline
\end{tabular}

Tabla 4. Niveles de nitratos en saliva población rural y urbana

\begin{tabular}{|lccc|}
\hline & Promedio & Desviación estándar & $p$ \\
\hline Rural & $19,7 \_\mathrm{g} / \mathrm{ml}$ & $35,5 \_\mathrm{g} / \mathrm{ml}$ & 0,17 \\
Urbana & $4,2 \_\mathrm{g} / \mathrm{ml}$ & $4,5 \_\mathrm{g} / \mathrm{ml}$ & \\
\hline
\end{tabular}


saliva, entre aquellos que presentaban estos factores con los que no lo presentaban.

Al realizar un análisis de correlación sólo se encontró significancia entre nivel de nitritos en saliva y nivel de nitratos en saliva $(p<0,01)$. No se evidenció correlación entre nivel de nitratos en agua y niveles en saliva $(p>0,05)$, como tampoco entre edad y niveles de nitratos y nitritos en grupo rural y urbano.

\section{DISCUSIÓN}

En la zona rural precordillerana hay niveles significativamente mayores de nitratos en el agua bebible comparado al agua potable de Concepción, siendo estos niveles superiores a normas nacionales e internacionales. Asimismo se encontró niveles elevados de nitratos ( $p$ $>0,05)$ y nitritos $(p<0,05)$ en la saliva de la población rural en comparación a la población urbana.

Según nuestros resultados, los niveles de sustancias nitrogenadas no fueron significativamente influenciados por el sexo, la edad, consumo de alcohol o tabaco. A pesar de no encontrarse una correlación estadísticamente significativa entre niveles en saliva y en agua, vale decir que a mayor nivel de nitratos en el agua no encontramos un mayor nivel estadísticamente significativo de nitritos y de nitratos en la saliva, sí existe una tendencia estadística, por lo que sería necesario ampliar el tamaño de muestra del grupo estudio para así tener una mayor potencia y de este modo establecer una relación con significancia estadística.

Debido a los resultados encontrados, y a los niveles considerablemente elevados, pensamos que el agua proveniente de napas de agua superficial no es apta para el consumo de la población.

Igualmente debiera existir en nuestro país, emulando a países con mayores estándares de control y regulación de la actividad agrícola, normativas en relación a la nitrificación de los cultivos, para así proteger las fuentes de agua de la contaminación por productos nitrogenados.

\section{CONCLUSIONES}

Los resultados confirman nuestra sospecha que los niveles de productos del ciclo del nitrógeno están elevados en el agua (NO3) proveniente de napas superficiales así como también lo están en la saliva (NO3 y N04) de las personas que la consumen en la población rural estudiada. Por lo tanto podrían jugar un rol en la génesis de los carcinomas escamosos oro e hipofaringe. Deben abrirse otras líneas de investigación para establecer su responsabilidad causal de esta enfermedad.

\section{Agradecimientos}

Este estudio clínico fue aprobado por el Comité de Ética del Hospital Clínico Guillermo Grant Benavente y forma parte de un Proyecto de Investigación concursado y financiado por la Sociedad Chilena de Otorrinolaringología, Medicina y Cirugía de Cabeza y Cuello.

Sr. Matías Hepp, Bioquímico, Facultad de Bioquímica de la Universidad de Concepción.

Sra. Maritza Flores, Bioestadística, Facultad de Medicina de la Universidad de Concepción.

\section{BIBLIOGRAFÍA}

1. Ribeiro KC, Kowalski LP, Latorre MR. Impact of comorbidity, symptoms, and patients' characteristics on the prognosis of oral carcinomas. Arch Otolaryngol Head Neck Surg 2000; 126:1079-85.

2. Ma N, Tagawa T, Hiraku $Y$, Murata $M$, Ding $X$, KaWANISHI S. 8-Nitroguanine formation in oral leukoplakia, a premalignant lesion. Nitric Oxide 2006; 14: 137-43.

3. Sutandyo N. Nutritional carcinogenesis. Acta Med Indones 2010; 42 (1): 36-42.

4. Tenovou J. The biochemistry of nitrates, nitrites, nitrosamines and other potential carcinogens in human saliva. J Oral Pathol 1986; 15: 303-7.

5. LIU RH, HотснкISS JH. Potential genotoxicity of chronically elevated nitric oxide: a review. Mutat Res 1995; 339: 73-89.

6. Reznick AZ, Hershrovich 0, Nagler RM. Saliva-a pivotal player in the pathogenesis of oropharyngeal cancer. Br J Cancer 2004; 91: 111-8.

7. Bahar G, Feinmesser R, Shpitzer T, Popovtzer A, Nagler R. Salivary analysis in oral cancer patients. Cancer 2007; 109: 54-9. 
8. Camargo JA, Alonso A. Ecological and toxicological effects of inorganic nitrogen pollution in aquatic ecosystems: A global assessment. Environment 2006; 32(6): 831-49.

9. Caballero Mesa JM, Rubio Armendáriz C, Hardisson DE LA ToRRE A. Nitrrate intake from drinking water on Tenerife island (Spain). Sci Total Environ 2003; 302(1-3): 85-92.
10. Even S, Billen G, Baco N, Théry S, Ruelland D, Garnier J, Cugier P, Poulin M, Blanc S, Lamy F, PAfFONI C. New tools for modelling water quality of hydrosystems: an application in the Seine River basin in the frame of the Water Framework Directive. Sci Total Environ 2007; 375(1-3): 274-91. 